\title{
Functional Characteristics of the Mouse //1b Promoter in Various Tissues Before and After Irradiation
}

\author{
Dmitriy Karpenko, Alyona Dorofeeva, Natalia Petinati, Irina Shipounova, Nina Drize, and Alexey Bigildeev
}

\begin{abstract}
Interleukin-1 beta $(I L I B)$ is a key inducer of inflammation and an important factor in the regulation of hematopoietic stem cells and mesenchymal stromal progenitors. Irradiation of mice with ionizing radiation has been shown to induce a lasting increase in IL1B concentration in peripheral blood. One of the possible mechanisms may be demethylation of $\mathrm{CpG}$ cytosines in the $I l 1 \mathrm{~b}$ promoter, which has not been characterized in detail for the mouse. In this study, the methylation level of CpGs located in a region between -3562 and -208 bp upstream of the start of transcription is studied in muscles, bones, liver, thymus, spleen, bone marrow, lymph nodes, lungs, and brain. The methylation level is compared to $I l l b$ expression. Tissue-specific features of $\mathrm{CpG}$ methylation are established. It is demonstrated that the region between -2420 and $-2406 \mathrm{bp}$ is likely a part of the mouse $111 \mathrm{~b}$ promoter/enhancer and may determine the base level of $I l l b$ expression in various tissues. Irradiation at a dose of $6 \mathrm{~Gy}$ does not change the methylation profile of most studied CpGs, and therefore, the cause of the stably increased IL1B level after irradiation is unlikely to be a change in the methylation of the studied CpGs in investigated tissues.
\end{abstract}

Keywords: $I l l b$ promoter, $\mathrm{CpG}$ methylation, irradiation, $I l l b$ expression

\section{Introduction}

$\mathbf{I}_{\mathrm{f}}$ NTERLEUKIN-1 $\beta(I L 1 B)$ IS A PROTOTYPE of the IL-1-like family of cytokines, which plays a key role in the induction of inflammation (Dinarello, 2013). Recent studies have shown that IL1B exerts other functions. For example, it directly regulates hematopoietic stem cells, stimulates the growth of bone marrow mesenchymal precursor cells, and increases their ability to support hematopoietic progenitor cells (Bigildeev et al., 2015; Pietras et al., 2016). The main source of IL1B in the body is activated macrophages in response to stress, neuroactive substances, cytokines, lipopolysaccharides, and other stimuli (Dinarello, 1996). Ionizing radiation can also activate the production of IL1B: it has long been known that the concentration of IL1B rises in the peripheral blood and other tissues after exposure to high doses of ionizing radiation (Johnston et al., 1996; Zhou et al., 2001; Linard et al., 2003, 2004). This rise is usually regarded as a marker of tissue damage. The results of our own experiments and data from other researchers indicate that irradiation leads not only to a short-term burst of IL1B but also to prolonged activation of its expression, which is measured in the peripheral blood and other organs and tissues for several months after irradiation (Johnston et al., 1996; Bigildeev et al., 2013). One possible explanation for this observation may be activation of the promoter of this gene. For a number of genes, including human $I L 1 B$, it has been shown that demethylation of cytosines in $\mathrm{CpG}$ islands located within the promoter is associated with activation of the expression of the corresponding gene, and vice versa; methylation of such cytosines is associated with a decrease in transcription (Fitzpatrick et al., 1998; Hashimoto et al., 2009, 2013; Ilnytskyy and Kovalchuk, 2011; Jones, 2012; de Araújo-Souza et al., 2015). The genes of the human IL1B and the mouse $I l 1 b$ were cloned in 1984 and 1986, respectively (Auron et al., 1984; Gray et al., 1986). These orthologous genes encode proteins that share $67 \%$ homology. The promoter of the human $I L 1 B$ and the mouse $I l 1 b$ genes has been studied in detail (Clark et al., 1986; Shirakawa et al., 1993; Godambe et al., 1994a, 1994b, 1995a). The human $I L 1 B$ promoter is known to include the proximal and distal parts (Shirakawa et al., 1993; Kominato et al., 1995) and ranges from -3757 to $+13 \mathrm{bp}$ relative to the transcription start site (TSS). The specified area contains 37 CpGs. Several transcription factors are known to have binding sites in the $I L 1 B$ promoter: for example, factors of the CEBP family (CEBPB, CEBPG), NF-kB (NFKB1, NFKB2, IKBA), AP-1 (FOS-JUN), PU.1 (SPI1), and others (Shirakawa et al., 1993; Kominato et al., 1995; Hashimoto et al., 2009). The proximal promoter, as well as the distal promoter regions, has been established as important for ILIB expression (Shirakawa et al., 1993; Tsukada et al., 1994; Hashimoto 
et al., 2009). Treatment of a chondrocyte culture with the demethylating agent 5-azacytidine, IL1B, or TNF $\alpha$ has been shown to decrease $\mathrm{CpG}$ methylation at position -299 , which is accompanied by a significant increase in the amount of ILIB mRNA (Hashimoto et al., 2009). Deletion mutagenesis has shown that the distal region of the promoter is also relevant for $I L 1 B$ expression. After removing nucleotides located between -2894 and $-2729 \mathrm{bp}$ upstream of TSS, there was a significant decrease in the expression of the reporter gene chloramphenicol acetyltransferase. This region includes CEBP (NF-IL6) and NF- $\beta 1 / \beta 2$ binding sites located between -2894 and -2831 bp (Shirakawa et al., 1993). Another independent distal promoter/enhancer element was defined between -2782 and -2729 bp of the human $I L 1 B$ to contain CEBP and CREB binding sites (Tsukada et $a l .$, 1994). The mouse $I l l b$ promoter was examined in a number of studies (for review, see Godambe et al., 1995b). In an early study of the structure of the mouse $I l l b$ gene, the nucleotide sequences located in regions between -195 and $-176 \mathrm{bp}$ and between -430 and $-419 \mathrm{bp}$ are analogous with sequences in human ILIA gene, from which it is concluded that these regions may be part of the mouse $I l 1 b$ regulatory sequence (Telford et al., 1986). This was proven in a seminal study of Godambe et al. They identified NFIL-6 (CEBP) binding site between -110 and -102 bp (coordinates used by Godambe were changed here and further in the text of this study by $-18 \mathrm{bp}$ according to the current knowledge) in $5^{\prime}$ flanking sequence of murine $I l l b$ TSS (Godambe et al., 1994b). This sequence was important for conferring lipopolyssacharide (LPS)-responsive activation of $I l 1 b$ expression in murine macrophage cell line RAW264.7. In the same study it was noted that a distal sequence located between -4111 and -2129 bp also contains regulatory elements of $I l l b$ transcription. The coordinates of the distal parts of murine $I l l b$ promoter were defined in the subsequent studies. It was established that there are at least two binding sites of transcription factors within the region between -2600 and $-2200 \mathrm{bp}$. The first one (NFIL-6-like) is located between -2253 and -2248 bp and it is responsible for binding CEBP proteins (Godambe et al., 1994a). The second one is located at $-2303 \ldots-2289$ (IL1 $\beta$-UNF1) and is responsible for binding unknown transcription factor (Godambe et al., 1995a). In this study, we evaluated the functional characteristics of the mouse $I l 1 b$ promoter and monitored the changes in the methylation profile of CpGs, located in the extended region upstream of TSS, after irradiation. A new potential element of $I l l b$ distal promoter/enhancer was identified as located between -2420 and $-2406 \mathrm{bp}$ upstream of TSS. The expression level of $I l 1 b$ in various tissues of nonirradiated mice was also determined and correlated with the level of CpG methylation in its promoter.

\section{Materials and Methods}

\section{Animals}

Female F1 hybrids $(\mathrm{CBA} \times \mathrm{C} 57 \mathrm{Black} / \mathrm{J} 6) \mathrm{F} 1$, aged 5 months to 2 years, obtained from the Stolbovaya breeding facility were used in the study. The animals were kept on $12 \mathrm{~h}$ of daylight and received food and water without restrictions. The experiments were approved by the Commission on Biomedical Ethics at the Institute of Biomedical Problems of the Russian Academy of Sciences (protocol no. 257).

\section{Irradiation}

Mice were exposed to $\gamma$-radiation from a ${ }^{60} \mathrm{Co}$ source at the "Luch-1" installation in the Tsyb National Medical Research Radiological Center, a branch of the Federal State Budgetary Institution "Scientific Medical Research Center of Radiology" of the Ministry of Health of the Russian Federation (Obninsk, Russia) at a dose of $6 \mathrm{~Gy}$ at a dose rate of $417 \mathrm{mGy} / \mathrm{min}$ and a focal length of $100 \mathrm{~cm}$.

\section{$D N A$ isolation}

DNA was isolated from muscles, bones, liver, thymus, spleen, bone marrow, and lymph nodes of control nonirradiated and irradiated mice. DNA from lungs and brain was isolated only for nonirradiated old animals. The bone marrow was washed with phosphate-buffered saline (PBS) from the cavities of the femora and tibia and resuspended into a single-cell suspension. Muscles and bones cleared of concomitant tissues were frozen in liquid nitrogen and triturated in a ceramic mortar with Sol2 lysis buffer (50 mM Tris-HCl, pH 8.0 [MP Biomedicals, United States], 2\% sodium dodecyl sulfate [MP Biomedicals], 2 mM EDTA, pH 8.0 [Sigma, United States]). Fragments of spleen, liver, thymus, lymph nodes (axillary, mesenteric, inguinal, and Peyer's patches), lungs, and brain were crushed in PBS in a glass Potter homogenizer. Cell suspensions were washed twice with PBS, and the cells were lysed with Sol2. DNA was isolated according to a standard method with proteinase $\mathrm{K}$, followed by extraction with $\mathrm{NaCl}$ and precipitation in ethanol. The obtained DNA was dissolved in TE buffer (10 mM Tris-HCl, pH 7.4, 1 mM EDTA, pH 8.0). For all the tissues except for lungs and brain, DNA was isolated from irradiated and nonirradiated animals. Lungs and brain were investigated in nonirradiated animals only.

\section{Bisulfite DNA conversion}

Bisulfite DNA conversion was performed using a commercial EZ DNA Methylation-Gold Kit (Zymo Research, United States) according to the manufacturer's instructions. For the reaction, $500 \mathrm{ng}$ of DNA of each sample was used. During the conversion, unmethylated cytosines are replaced with uracils, which in turn are replaced by thymidines as a result of subsequent PCR.

\section{Conventional PCR}

Converted DNA was used for PCR of the areas of interest in the regulatory region of the $I l l b$ gene. The primer sequences and reaction conditions are described in Supplementary Table S1. PCR was performed in a Tertsik thermocycler (DNA Technology, Russia). As a rule, the following parameters were used: preheating to $95^{\circ} \mathrm{C}$ for $10 \mathrm{~min}$, cyclic denaturation at $95^{\circ} \mathrm{C}$ for $30 \mathrm{~s}$, hybridization of primers at $53-65^{\circ} \mathrm{C}$ for $30 \mathrm{~s}$, elongation of the chain at $72^{\circ} \mathrm{C}$ for $60 \mathrm{~s}$, and final elongation at $72^{\circ} \mathrm{C}$ for $5 \mathrm{~min}$. Determination of the nucleotide sequence of the PCR products was carried out with Applied Biosystems 3730 DNA Analyzer (Thermo Fisher, United States) at the "Genome" Core Facility Center.

\section{Agarose gel electrophoresis}

The PCR results were evaluated by agarose gel electrophoresis containing $1-2 \%$ agarose (Sigma) and $0.0025 \%$ 
ethidium bromide (Merck, Germany). A fragment containing the PCR product of the correct length was cut out of the gel, and DNA was isolated using Wizard SV Gel and PCR Clean-Up System Kit (Promega, United States).

\section{Cloning conversion products}

The purified PCR products were ligated into pGEM-T Easy vector (Promega). Competent Escherichia coli strain XL-1 Blue cells were transformed with the vector. Colonies containing the inserts were selected according to the blue/ white test. For each DNA sample obtained from bones, 12 to 54 clones were collected. DNA was isolated from the colonies by boiling for a short time. The resulting DNA samples were used as templates for PCR with M13 primers (Supplementary Table S1). In some cases, the PCR product was purified from the components of the PCR mixture using Wizard SV Gel and PCR Clean-Up System columns without electrophoresis, and the nucleotide sequence of purified DNA was determined using one of the M13 primers.

\section{Analysis of the nucleotide sequence of mouse $/ 11 \mathrm{~b}$ and human IL1B promoters}

NCBI Reference Sequence NM_008361.4 was used to establish the boundaries of the mouse $I l 1 b$ mRNA. It was plotted to NCBI Mus musculus strain C57BL/6J chromosome 2 sequence (NCBI Reference Sequence NC_000068.7, GRCm38.p6 C57BL/6J) to derive the sequence of 5'flanking region of the promoter of mouse $I l 1 b$ gene. Nucleotide sequences with coordinates -3757 to $+13 \mathrm{bp}$ from TSS of the human and mouse genes encoding IL1B were compared using the Align tool of Vector NTI Advance software v. 1.5.3 (Invitrogen, United States). The nucleotide sequence of the mouse $I l l b$ promoter was examined for the presence of transcription factor binding sites using the online database HOCOMOCO (Kulakovskiy et al., 2016). As a measure of the reliability of determining the binding site, a confidence level of $p=0.0001$ was chosen.

\section{Determination of CpG methylation level in clones}

The degree of $\mathrm{CpG}$ methylation in clones of the PCR product was performed for DNA samples obtained from bones. The resulting nucleotide sequences were analyzed using online BISMA tool of the BDPC DNA methylation analysis platform. An example of the analysis result is shown in Supplementary Figure S1. The degree of cytosine methylation in each $\mathrm{CpG}$ was determined as a ratio of clones in which cytosine was methylated at this position. To calculate the error, unmethylated bases were assigned the conditional value " 0 " and methylated bases, " 1 " The mean value for each $\mathrm{CpG}$ and the standard error of the mean were calculated. The significance of the difference from the control sample was determined using Student's $t$-test.

\section{Determination of CpG methylation level in total PCR product}

Files with sequencing results were processed using Seq Scanner 2 (Applied Biosystems, United States) and SnapGene Viewer (GSL Biotech LLC, United States) software. The proportion of unmethylated cytosines at each $\mathrm{CpG}$ position in the total PCR product was determined by the am- plitude of the thymidine signal (" $T$ ") in the corresponding position. The calculation algorithm was as follows. The amplitude of the thymidine peak was measured at the $\mathrm{CpG}$ position (" $T_{0}$ ") and to the left and to the right of $T_{0}$ (" $T_{-}$", and " $T_{+}$," respectively) (Supplementary Fig. S2). The value $M_{-}^{s}$, calculated by the equation:

$$
M_{-}^{s}=\frac{T_{0}}{\frac{T_{-}+T_{+}}{2}},
$$

could serve as an indicator of the extent of demethylation for each cytosine. However, to improve the accuracy of calculations, the values of $T_{0}, T_{-}$, and $T_{+}$were also measured in the nucleotide sequence of a clone $\left(T_{0 \mathrm{c}}, T_{-\mathrm{c}}\right.$, and $\left.T_{+\mathrm{c}}\right)$ obtained from the total PCR product, which was sequenced with the same automatic analyzer at the same time. The clone was chosen such that the cytosine in the selected $\mathrm{CpG}$ position was unmethylated and, therefore, a thymidine signal was exclusive in the $T_{0 \mathrm{c}}$ position. The normalized $M_{-}^{n}$ value was calculated by the equation:

$$
M_{-}=\frac{\frac{T_{0}}{T_{0 c}}}{\frac{\frac{T_{-}}{T_{-c}}+\frac{T_{+}}{T_{+c}}}{2}}
$$

The resulting value was used to estimate the proportion of unmethylated cytosines.

\section{Calculation of the method error for determining CpG methylation in total PCR product}

To calculate the error of the method, the value of $D_{i}=\frac{T_{-}}{T_{+}}$ was calculated, where the index $i$ denotes the $i$ th DNA sample. The average $\bar{D}$ value for each $\mathrm{CpG}$ was calculated for all DNA samples, regardless of tissue or exposure. Next, the relative measurement error of $D_{i}$ was determined by the standard equation:

$$
\sigma_{D_{i}}=\left|\frac{D_{i}-\bar{D}}{\bar{D}}\right|
$$

To determine the relative measurement error of the proportion of unmethylated cytosines at each $\mathrm{CpG}$ position, the root-mean-square relative error was calculated for each sample using the equation:

$$
\sigma_{M_{-i}}=\sqrt{\left(\frac{\delta}{T_{-i}}\right)^{2}+\left(\frac{\delta}{T_{+i}}\right)^{2}+\left(\frac{\delta}{T_{0 i}}\right)^{2}+{\sigma_{D_{i}}}^{2}+{\sigma_{c}}^{2}}
$$

where $\delta$ is the systematic absolute error in determining the amplitude of the thymidine signal, and $\sigma_{c}$ is the relative error associated with determining the amplitudes of the 
signals in the normalization sample of a clone (it can be neglected). The error $\sigma_{M_{-i}}$ is indicated in Figures 2 and 3. It does not take into account the error associated with the individual biological characteristics of experimental animals.

Upon averaging the extent of $\mathrm{CpG}$ demethylation over the group of DNA samples $\left(\overline{M_{-G}}\right)$, the root-mean-square error was calculated:

$$
\sigma_{M_{-G}}=\sqrt{\sum_{i}^{G} \frac{\sigma_{M_{-i G}}{ }^{2}}{N(N-1)}},
$$

where $G$ denotes the averaged group, $N$ is the number of samples in the group, $\sigma_{M_{-i G}}$ is the relative error of $M_{-i}$ in the group, calculated by the equation:

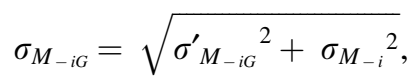

where $\sigma_{M_{-i G}}$ is the relative measurement error of $M_{-i}$ of the average value of $M_{-}$over a group of samples calculated by the equation:

$$
\sigma_{M_{-i G}}^{\prime}=\left|\frac{M_{-i G}-\overline{M_{-G}}}{\overline{M_{-G}}}\right| .
$$

The error $\sigma_{M_{-G}}$ from the Equation (5) is indicated in Figure 4. It takes into account both the calculation error and the error associated with biological replicates.

\section{$R N A$ isolation and reverse transcription}

To obtain cell lysates of the bone marrow, spleen, thymus, liver, and lymph nodes, the same procedures were performed as for DNA isolation, but instead of Sol2 lysis buffer, DR buffer was used $(25 \mathrm{mM}$ sodium citrate [Sig$\mathrm{ma}$, $0.5 \%$ N-lauryl sarcosine [ICN Biomedicals, United States], $4 \mathrm{M}$ guanidine thiocyanate [MP Biomedicals], and $0.1 \mathrm{M} \beta$-mercaptoethanol [ICN Biomedicals]). Total RNA was isolated according to Chomczynski with modifications as described (Chomczynski and Sacchi, 2006; Bigildeev et al., 2017). To isolate RNA from bones and muscles, the same steps were performed as for DNA extraction, but TRIzol reagent (Ambion, United States) was used as a lysis buffer. The concentration of RNA and its purity were evaluated by the absorption at $260 \mathrm{~nm}$ and the ratio of A260/A280, respectively. For bones and muscles, an additional quality control for RNA was performed using denaturing agarose gel electrophoresis (Sambrook et al., 1989). cDNA synthesis was carried out in two steps. First, a mixture of T13 primers and random hexamers (Sintol, Russia) was hybridized to RNA. In the second step, reverse transcription was performed using Moloney Murine Leukemia Virus (M-MLV) reverse transcriptase (Promega) in the required amount of buffer (Promega) with the addition of RNAsin (Promega) RNAse inhibitor to the sample. The reaction was carried out by incubating the samples at $42^{\circ} \mathrm{C}$ for an hour.

\section{Real-time PCR}

The level of $I l 1 b$ expression was determined using realtime PCR (TaqMan modification) on a StepOnePlus ${ }^{\mathrm{TM}}$ RealTime PCR System (Thermo Fisher Scientific, United States). The set and number of the most suitable reference genes were determined using GeNorm v. 3.4 software (Vandesompele et al., 2002). The search for optimal reference genes was carried out among nine genes (Sdha, Tbp, Ubc, Rpl13a, B2m, Hprt1, Actb, Hmbs, and Gapdh). For each cDNA sample, the expression level of $I l l b$ was determined relative to each of the selected reference genes as $2^{\Delta C_{q_{i}}}=2^{-\left(C_{q}^{I l 1 b}-C_{q}^{H K_{i}}\right)}$, where $C_{q}^{I l 1 b}$ is a quantitation cycle for $I l l b$, and $C_{q}^{H K_{i}}$ is a quantitation cycle for the $i$-th reference gene. The obtained values were averaged for samples $(n=3)$ of each tissue. The ratio normalized by the $i$-th reference gene $I l l b$ expression in each $j$-th tissue to the bone marrow was calculated as $R_{i j}=\frac{\left\langle 2^{\Delta C_{t_{i}}^{j}}\right\rangle}{\left\langle 2^{\Delta C_{t_{i}}^{\mathrm{KM}}}\right\rangle}$. Thereafter, the geometric mean $\left\langle R_{j}\right\rangle$ was calculated for all $i$ reference genes to determine the normalized value of $I l l b$ expression in each $j$-th tissue relative to the bone marrow. The calculation error of the obtained value was determined as the result of raising 10 to a power equal to the standard error of the arithmetic mean of $\log _{10}\left(R_{i j}\right)$. The statistical significance of the differences was assessed by the Mann-Whitney test applied to $\log _{10}\left(R_{i j}\right)$.

\section{Statistical analysis}

Statistical differences in CpG methylation between control nonirradiated and irradiated samples at each time point after exposure were evaluated by Student's $t$-test for PCR clones and by Mann-Whitney test for the total PCR products. Differences in relative gene expression level between control tissues were evaluated by Mann-Whitney test. A level of $p=0.05$ was set to be the cutoff for statistical significance.

\section{Results}

\section{Choosing CpGs for analysis}

Comparison of the regions upstream of TSS between human and mouse DNA showed that they coincide by $47.7 \%$. Based on data on the mouse $I l l b$, we decided to explore a region between -3562 and -208 bp in the mouse DNA to determine additional components of the promoter, establish the $\mathrm{CpG}$ methylation profile in various tissues, and investigate possible changes at different time points after irradiation of mice. The chosen area includes $22 \mathrm{CpG}$ dinucleotides (Fig. 1). Three CpG groups were selected for the analysis of methylation profile. The selection criterion was their grouping on a relatively short interval of up to $400 \mathrm{bp}$ and an approximate overlap of the positions important for the activity of the human $I L 1 B$ promoter.

\section{CpG methylation profile of the mouse II1b promoter in bones after irradiation}

The analysis was performed both for the total PCR product obtained after bisulfite conversion of DNA and for 1050 randomly selected copies of the total product obtained by cloning in E. coli (Fig. 2 and Supplementary Data S1). 


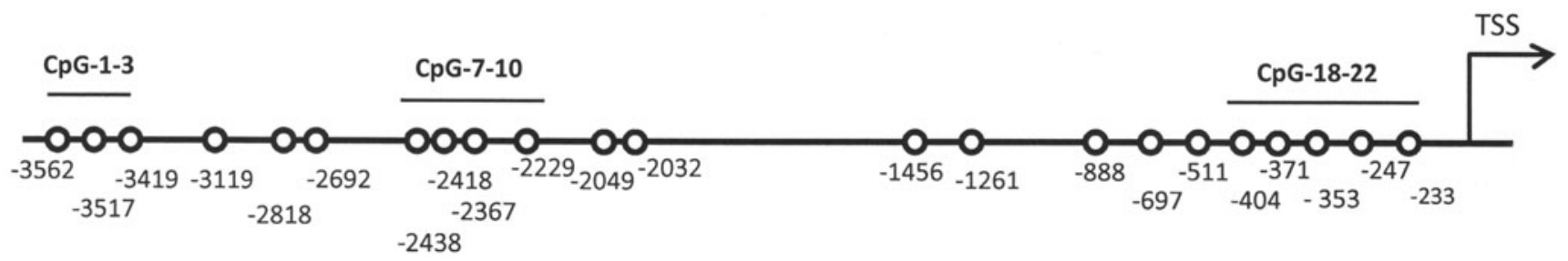

FIG. 1. Schematic representation of the regulatory region of the mouse $I l 1 b$, indicating the positions of CpG dinucleotides relative to the TSS. The horizontal bars represent the areas selected for analysis of the CpG methylation profile. Circles represent individual $\mathrm{CpGs}$, and the numbers indicate the $\mathrm{CpG}$ coordinates relative to the TSS. The arrow designates TSS. TSS, transcription start site.
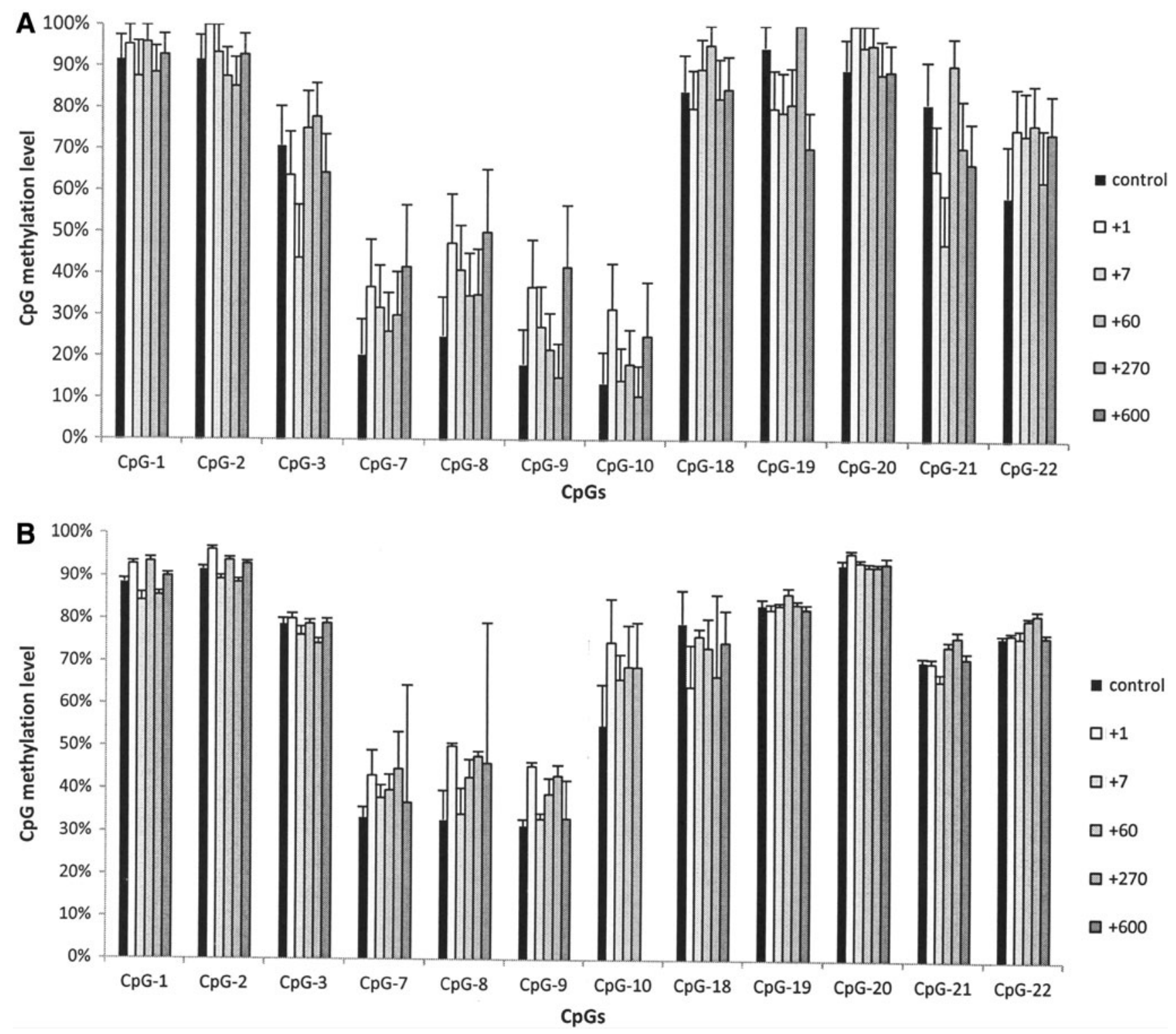

FIG. 2. Methylation profile of selected CpGs in the putative promoter of $I l l b$ in nonirradiated bones and at different time points after irradiation. Black bars represent nonirradiated controls; gray gradient bars represent data obtained at $+1,+7$, $+60,+270$, and +600 days after irradiation of mice. Each time point represents one animal. Errors are calculated as described. Methylation level is presented as a percentage of $\mathrm{CpG}$ methylation, where 100\% correspond to fully methylated cytosine, and $0 \%$ corresponds to fully nonmethylated one. CpG numbers follow Fig. 1. (A) Data on the PCR product clones. Each bar represents mean value calculated for $n=10-50$ individual clones. (B) Data on the total PCR product without cloning. See Supplementary Data S1 for raw data used for this figure. 
Both methods showed that the least methylated $\mathrm{CpGs}$ were CpG-7 to CpG-9 located between -2543 and -2206 bp upstream of TSS. No significant differences were found between irradiated and nonirradiated bones as assessed either by clones or total PCR product at any time point after irradiation. Analysis of $I l 1 b$ expression in bones 450 days after irradiation did not reveal differences from the control (data not shown), which is consistent with the lack of changes in the CpG methylation.

\section{CpG methylation profile in the mouse $\mathrm{Il} 1 \mathrm{~b}$ promoter in various tissues after sublethal irradiation}

For muscles, liver, thymus, spleen, bone marrow, and lymph nodes, the methylation profile was evaluated in the total PCR product obtained after DNA conversion of control and irradiated animals (Fig. 3 and Supplementary Data S2 and S3). It was noted that in the spleen and lymph nodes, unlike bones, a decrease in CpG-20 methylation was observed a day after irradiation. Changes in CpG-20 methylation were also observed at other time points after irradiation in the bone marrow (increase at +1 and +600 days), liver (decrease at +60 and +600 days), and muscles (decrease at $+7,+60$, and +270 days). Except for the described changes, irradiation at a dose of $6 \mathrm{~Gy}$ did not alter the methylation profile of investigated $\mathrm{CpGs}$ in the mouse $I l 1 b$ promoter. This allowed us to group samples by tissue, regardless of the irradiation and the elapsed time, and to compare the methylation profile among different tissues.

\section{Features of the CpG methylation profile in the mouse II1b promoter in various tissues}

A comparison of the studied CpGs among the tissues revealed interesting features of their methylation profile (Fig. 4 and Supplementary Data S4). It was shown that three $\mathrm{CpGs}$ located in the most distal region were almost completely methylated in all the studied tissues. CpG-18-CpG22 , located proximal to the TSS region (including CpG-20 at -353 position), were partially demethylated, but it was not possible to establish statistically significant differences between the tissues. The most differentially methylated sites were CpG-7-CpG-9.

\section{Expression of $1 / 1 \mathrm{~b}$ in tissues}

Since it is known that the methylation extent of individual CpGs in the promoter of human $I L 1 B$ significantly affects the level of transcription, we decided to compare the expression of $I l l b$ with $\mathrm{CpG}$ methylation in its regulatory region in tissues of nonirradiated mice. When comparing gene expression in a wide range of tissues consisting of different cell types, it is very important to select reference genes whose expression varies minimally in the tissues studied. The choice of these genes significantly affects the results obtained. We conducted a thorough analysis of nine genes frequently used to normalize the results of gene expression experiments. It was found that in the present study, normalization needed to be performed with the seven most stable genes, which for our tissue set were $S d h a, T b p, U b c$, Rpl13a, B2m, Hprt1, and Actb. The results of the comparison of $I l l b$ expression across the tissues are shown in Figure 5 (see also Supplementary Data S5). The highest level of $I l 1 b$ expression was observed in the bone marrow, which is consistent with the fact that hematopoietic cells are the main producers of $I l 1 b$. It is interesting to note that CpG7-CpG-9 in bone marrow cells were maximally demethylated. The expression level in the spleen was lower than in the bone marrow, but the spleen, liver, and bones did not differ among themselves. We compared our results with the expression atlas database of the ENSEMBLE project and with the data from the ENCODE project. The ENSEMBLE project contains transcriptome data analysis for bones, thymus, spleen, lung, and brain. According to that database, $I l 1 b$ expression shows the following decreasing order: lungs (32 TPM) $>$ spleen $(22 \quad$ TPM $)>$ bones $(11$ TPM $)>$ thymus (2 TPM) $>$ brain (0.5 TPM). At the same time, the lungs, spleen, and bones are comparable with each other and are characterized by relatively high levels of expression, and both the thymus and the brain are designated as the tissue with low level of $I l 1 b$ expression. Data on muscles and liver are presented only for young mice. These tissues are characterized by even lower level of expression than the thymus of adult animals (Expression Atlas database*). The database of the ENCODE project contains information on the spleen, thymus, and liver (NCBI database ${ }^{\dagger}$ ). According to that database, $I l 1 b$ expression in these tissues shows the following descending order: spleen (1.324 RPKM)>lungs (1.048 RPKM)>thymus (0.271 RPKM)>liver (0.095 RPKM)>brain (0.028 RPKM, mean for cerebellum and frontal lobe).

\section{Binding sites of transcription factors}

Three regions containing the examined CpGs were analyzed for the presence of transcription factor binding sites using the HOCOMOCO database. The search was performed for those factors, for which the binding sites in human ILIB promoter are known: SPI1 (PU.1), CEBPB, NFKB (NFKB1, NFKB2), and TBP. Of the three analyzed segments (Fig. 1), only the region located between -2543 and $-2206 \mathrm{bp}$ contained the binding sites of these transcription factors (Fig. 6).

\section{Discussion}

This study was inspired by an observation that the level of IL1B remains elevated for a long time in the peripheral blood of mice after sublethal irradiation. This effect cannot be explained by a transient inflammation in injured by radiation tissues, since it lasts from the day of exposure to death of the animal, which could happen several months after and is not related to irradiation. One possible explanation of the observed effect could involve changes in $\mathrm{CpG}$ methylation within promoter region of the gene. Since the mouse $I l l b$ promoter is at least partly characterized predominantly in macrophage cell lines (Godambe et al., 1994a, 1994b, 1995a), it is interesting to know what factors regulate $I l l b$ expression in the primary tissues and how transcription factors binding sites cooperate with $\mathrm{CpG}$

*https://www.ebi.ac.uk/gxa/genes/ensmusg00000027398?bs= $\% 7 \mathrm{~B} \% 22$ mus $\% 20$ musculus $\% 22 \% 3 \mathrm{~A} \% 5 \mathrm{~B} \% 22 \mathrm{ORGANISM} P$ PART $\% 22 \% 5 \mathrm{D} \% 7 \mathrm{D} \& \mathrm{ds}=\% 7 \mathrm{~B} \% 22 \mathrm{kingdom} \% 22 \% 3 \mathrm{~A} \% 5 \mathrm{~B} \% 22$ animals $\%$ $22 \% 5 \mathrm{D} \% 7 \mathrm{D} \#$ baseline

${ }^{\dagger}$ https://www.ncbi.nlm.nih.gov/gene/16176/?report=expression 

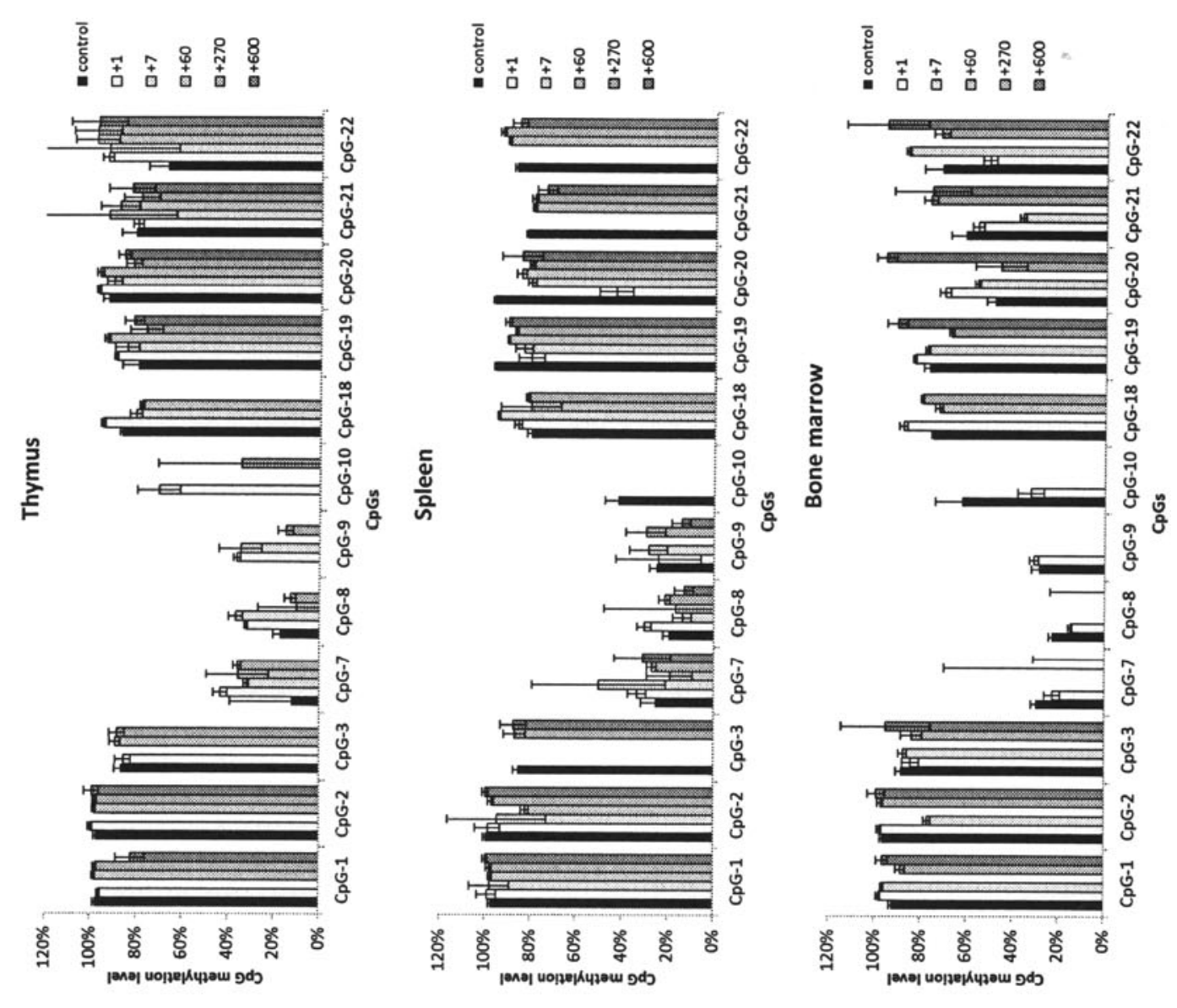

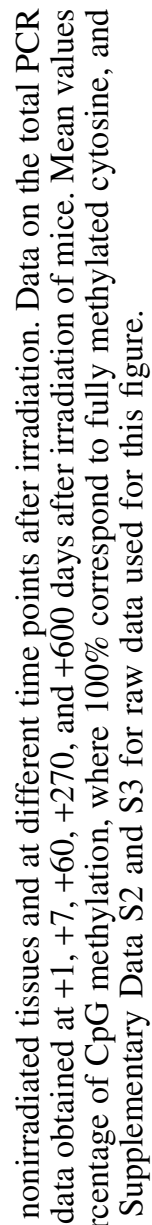

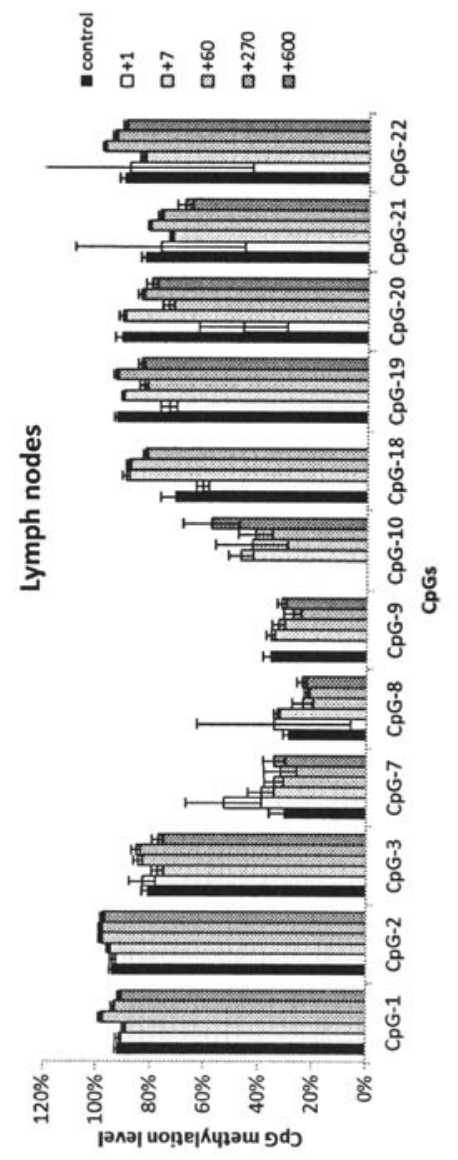

Iริ

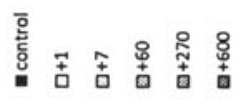
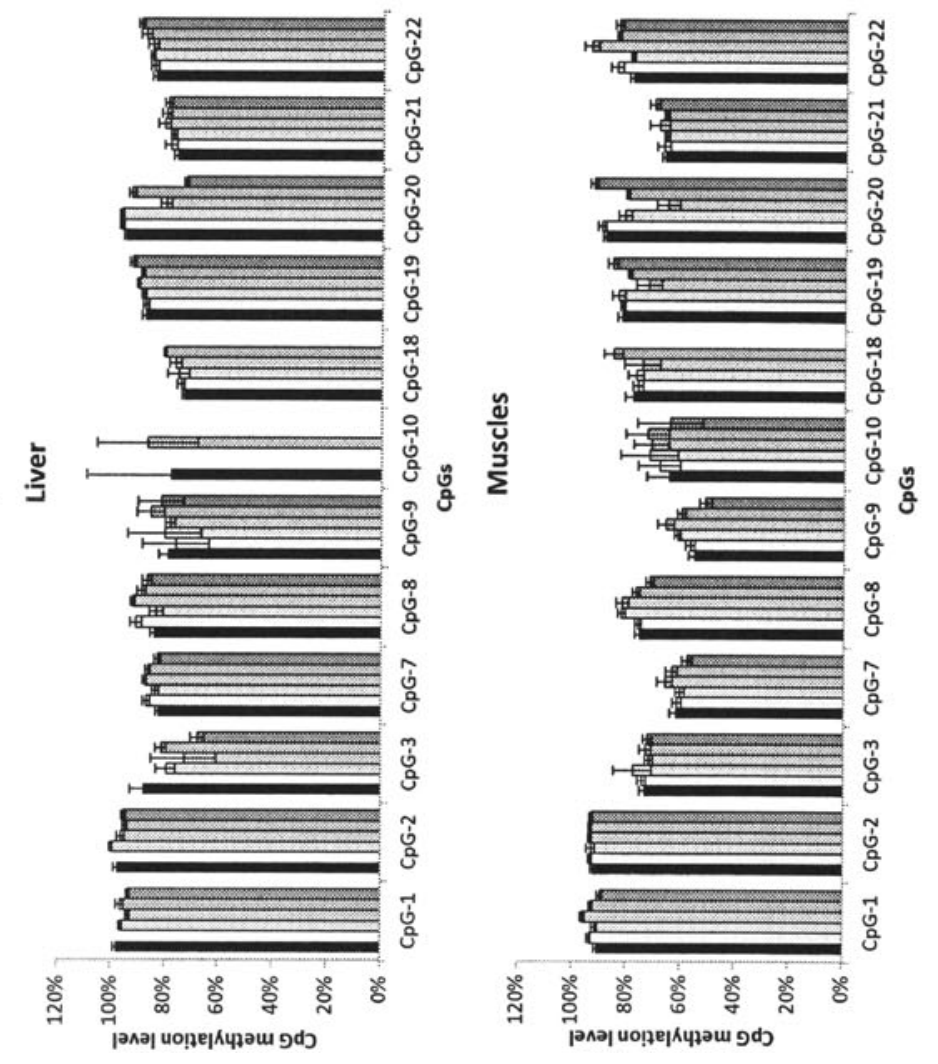

$\Xi$ 过

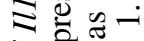

पै

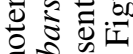

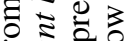

현응

$\geq \div$

츨

호응

的票

.

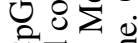

U형

可

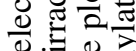

․․ㄹ

प워

늘 苞

훈즌

힌

बै

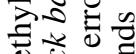

$\sum \frac{0}{0}$ 명

ம் 范

نำ

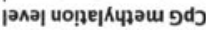

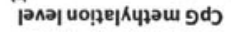

至范 


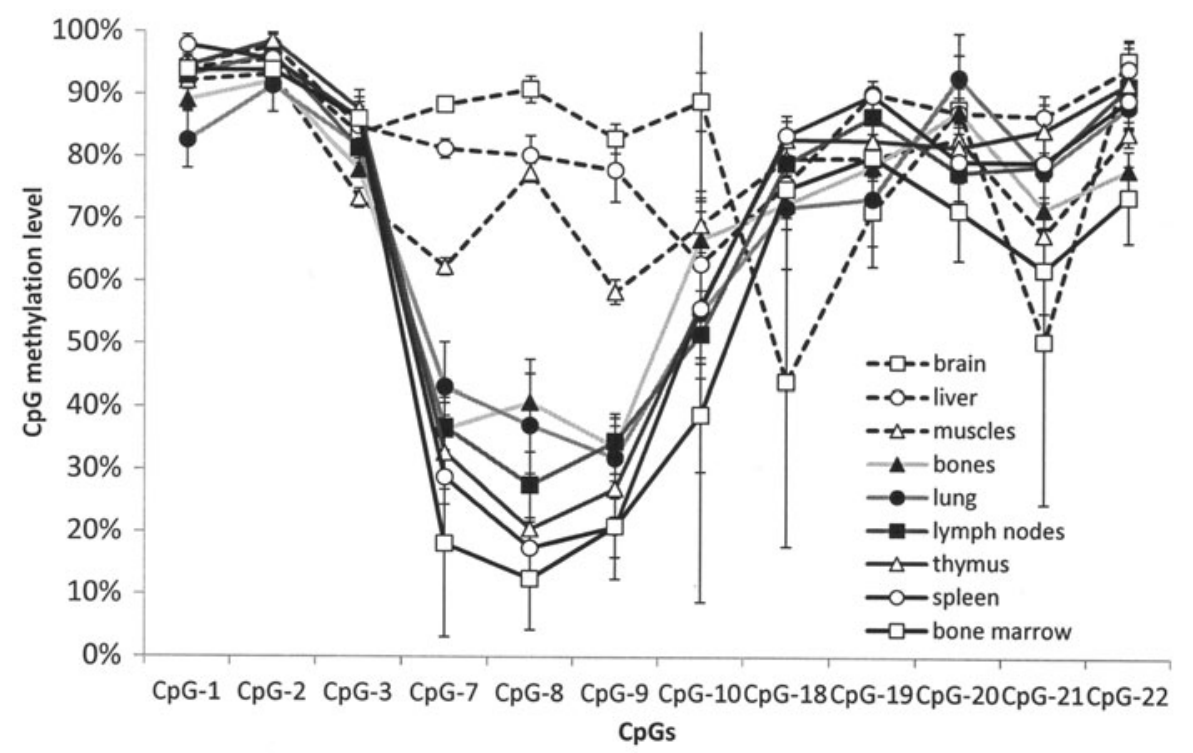

FIG. 4. Methylation profile of the putative $I l l b$ promoter region in mouse tissues. Mean values $(n=6)$ and standard error of the mean are plotted. Methylation level is presented as a percentage of $\mathrm{CpG}$ methylation, where $100 \%$ correspond to fully methylated cytosine, and $0 \%$ corresponds to fully nonmethylated one. $\mathrm{CpG}$ numbers follow Figure 1. See Supplementary Data S4 for raw data used for this figure. methylation within the promoter. To answer these questions we studied methylation level of CpGs in the mouse $I l 1 b$ promoter before and long after irradiation in different tissues and compared $I l l b$ expression level among them. Since the absence of $\mathrm{CpG}$ within the proximal part of the mouse promoter adjacent to TSS, we were mainly interested in the region located beyond $-200 \mathrm{bp}$ from TSS. Special attention was paid to the bones, since it was shown in earlier experiments in vitro and in vivo that the bones of irradiated mice secrete an increased amount of IL1B (Bigildeev et al., 2013). Two approaches were applied to DNA isolated from the bones of irradiated and control animals when analyzing CpG methylation profile. Both methods yielded a similar CpG methylation profile (Pearson correlation coefficient 0.83 ), which indicated the reliability of the developed method for the analysis of $\mathrm{CpG}$ methylation in total PCR product. No changes were detected in $\mathrm{CpG}$ methylation of the studied part of $I l l b$ promoter and in its expression 450 days after irradiation. The information obtained in this study differs from the results of one of the previous experiments, in which an increase in $I l 1 b$ expression in bone cells after irradiation at a similar dose was observed several months after exposure (Bigildeev et al., 2012). The observed inconsistencies are probably due to different experimental conditions. The lack of differences between irradiated and nonirradiated bones in this study allowed us to conclude that irradiation of mice at a dose of $6 \mathrm{~Gy}$ does not affect the methylation profile of the studied CpGs in the mouse $\mathrm{Illb}$ promoter in bones.

The analogous approach of measuring $\mathrm{CpG}$ methylation in total PCR product was applied to other investigated tissues of control nonirradiated and irradiated animals. We were able to find some deviations of CpG-20 methylation, for which direction, value, and time course depended on specific tissue. These changes indicate a potential role of CpG-20 at -353 bp in $I l 1 b$ regulation. However, except for the described variations, there were no differences in the methylation profile between the tissues of control and irradiated animals at any time point after irradiation (Fig. 3). This finding is consistent with the results of previous studies, where no changes in $I l 1 b$ expression in the bone marrow, spleen, thymus, and liver were observed 3 months after irradiation under similar experimental conditions. This implies that the source of prolonged elevated level of IL1B in the peripheral blood after irradiation can originate in other tissues. For example, it was shown in an earlier study that an increased level of $I l 1 b$ expression had been observed in lungs for several months after irradiation of mice at $12 \mathrm{~Gy}$

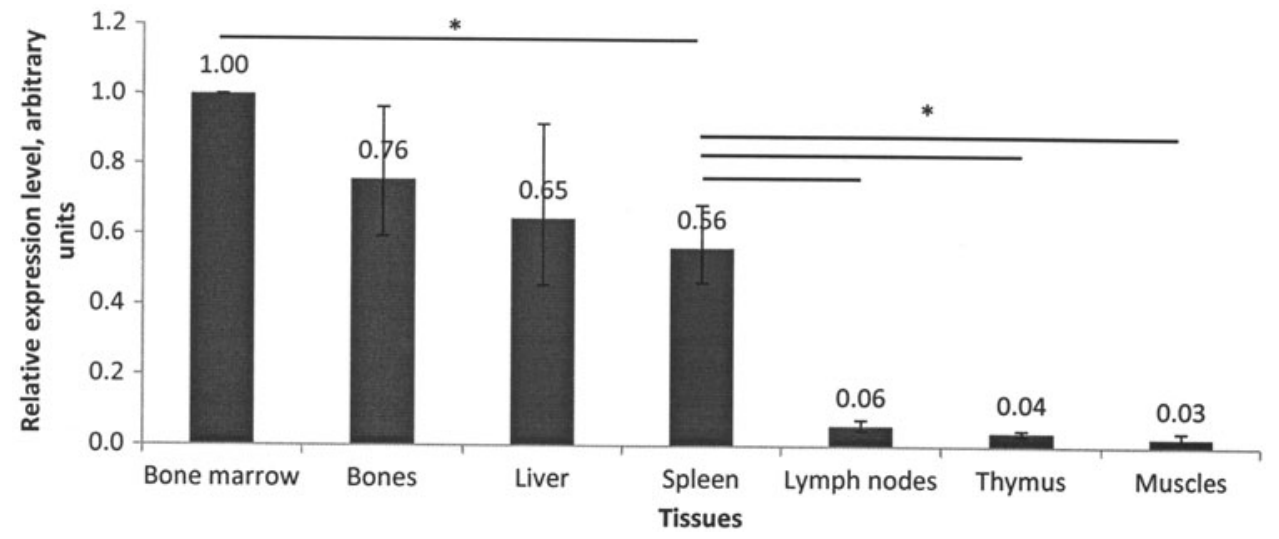

FIG. 5. Comparison of the $I l l b$ expression among tissues. The level of expression in the bone marrow is set to be 1 . Numbers indicate mean values $(n=3)$ for each tissue, and the standard error of the mean is plotted. Statistically significant differences between tissues are designated by asterisks with horizontal lines connecting corresponding tissues. See Supplementary Data S5 for raw data used for this figure. 
FIG. 6. The result of HOCOMOCO analysis of the region located between -2543 and $-2206 \mathrm{bp}$ from the TSS of the mouse Illb. Dark gray area indicates $\mathrm{CpG}$; gray area, SPI1, and CEBPB binding sites. As a measure of the reliability of determining the binding site, a confidence level of $p=0.0001$ was chosen.
CpG-7 CTGAGTGAGTCAAGAGCTGAAAAGTCCCCAAAAGCTAGAGTGCCCGTCACCATCCTGGCTTTG

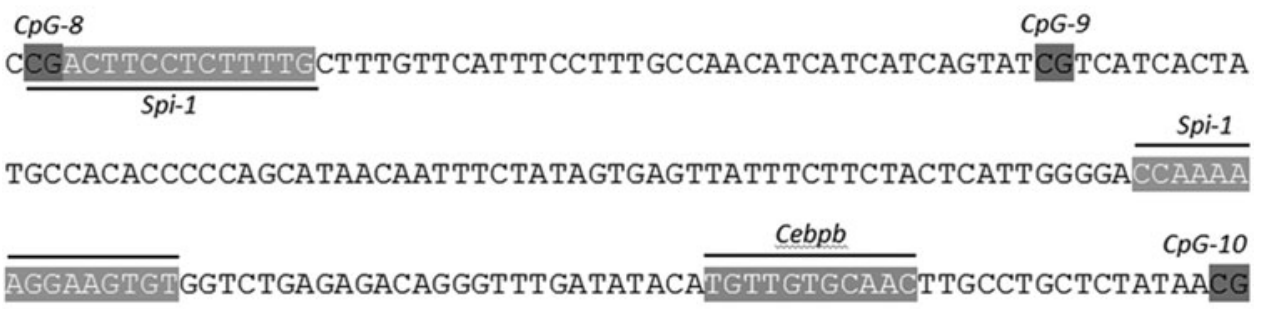

ACAAGGGGAGGAAATTTGGAGCCC

$-2206$

(Johnston et al., 1996). The absence of remarkable changes in $\mathrm{CpG}$ methylation within $I l 1 b$ promoter allowed us to group samples by tissue, regardless of the irradiation and the elapsed time, and to compare the methylation profile among different tissues.

The most differentially methylated sites appeared to be CpG-7-CpG-9. We assume that the region located between -2543 and -2206 bp upstream of TSS may be responsible for the differential expression of mouse $I l l b$ in the tissues. It is known for human $I L 1 B$ that the main sequences important for its differential expression are located in the intervals between -131 and $+12 \mathrm{bp},-2729$ and $-1097 \mathrm{bp}$, and between -1097 and -131 bp (Shirakawa et al., 1993). Thus, the regions responsible for the differential expression of $I L 1 B$ in humans and mice may partially overlap.

Apparently, all inspected tissues split into two groups. The first one included brain, liver, and muscles and could be characterized by high methylation level of CpG-7-CpG9. The second one included bones, lungs, lymph nodes, thymus, spleen, and bone marrow, which had relatively low methylation level of CpG-7-CpG-9. It is generally accepted that transcription of a gene is inversely related to $\mathrm{CpG}$ methylation level of its promoter. We asked if this is true for $I l l b$ and compared its expression among designated tissues with $\mathrm{CpG}$ methylation of the studied region in corresponding tissue. It is important to note that the comparison of gene expression among tissues, which substantially differ in their function and cell composition, is challenging. We tested nine potential reference genes and established that normalization in our experiment should be performed by at least seven most stable genes. The expression results revealed two groups, tissues with a relatively high expression, comparable to that in the bone marrow (bones, liver, and spleen), and tissues with low expression (lymph nodes, thymus, and muscles). We compared our data to two open databases of ENSEMBLE and ENCODE and found that the results obtained in our study are consistent with the ENSEMBLE and ENCODE data, with the exception of the liver, in which we observe an abnormally high level of $I l 1 b$ expression. It is possible that these differences are related to the biological charac- teristics of the mouse strain used in this study and to the age of the animals, which at the time of the study was 8 months.

The data on the expression and methylation of CpG-7CpG-9 are consistent in the bone marrow, where the maximum expression level and minimum methylation of the indicated CpGs were observed, and in the muscles, where low expression and high methylation were revealed. However, overall, the methylation level of CpG-7-CpG-9 did not reflect the $I l 1 b$ expression level in each specific tissue. This observation indicates that other CpGs located between -3562 and $+1 \mathrm{bp}$, which were not studied, may affect $I l 1 b$ expression level. Additional factors, such as different expression levels of transcription factors, their negative regulators, and post-translational modifications of histone proteins, may affect the baseline level of $I l l b$ expression in each specific tissue. For example, it is known that the tissuespecific activity of human $I L 1 B$ promoter is regulated by the transcription factor SPI1 (PU.1); in the absence of this factor, the promoter is not active (Kominato et al., 1995). Taking into account these considerations, we analyzed the region between -3562 and +1 bp upstream of murine $I l 1 b$ TSS for the binding sites of transcription factors, which are known to bind to human $I L 1 B$ promoter, such as SPI1 (PU.1), CEBPB, NFKB, and TBP. It is interesting that CpG8 , one of those CpGs whose methylation level differed among tissues, overlaps with the binding site of SPI1 transcription factor, which in turn determines the tissue-specific activity of the $I L 1 B$ promoter. This finding suggests that the region between -2420 and $-2406 \mathrm{bp}$ is also a functional part of the mouse $I l l b$ promoter/enhancer and that the basal level of $I l l b$ expression in a tissue is determined by a combination of at least two factors: the methylation level of $\mathrm{CpG}$ at -2420 bp position and the expression level of Spil in that tissue. This multifaceted regulation may account for the lack of correlation between the methylation level of examined CpGs and the $I l l b$ expression in the studied tissues.

\section{Conclusion}

Our study demonstrated that irradiation of mice at $6 \mathrm{~Gy}$ did not lead to sustained changes in the methylation profile 
of CpGs located within the proposed $I l 1 \mathrm{~b}$ regulatory region in bones, bone marrow, spleen, thymus, lymph nodes, liver, and muscles. Therefore, the mechanism for the stably elevated level of IL1B in the peripheral blood of irradiated mice is not associated with (de)methylation of the studied part of mouse $I l l b$ promoter in the examined tissues. The results suggest that the source of long-term production of IL1B should be sought in other tissues. However, the results of this study do not exclude the possibility that significant changes in $\mathrm{CpG}$ methylation and $I l l b$ expression occur in one or several of the examined tissues, but only in a small subpopulation of cells. As a result, the sensitivity of applied methods may not be sufficient to reveal these differences in the total cell populations of organs and tissues. To achieve this, it is necessary to conduct research at the level of RNA and DNA sequencing of individual cells. The results of the study show that the region located between -2420 and $-2406 \mathrm{bp}$ from TSS of the mouse $I l 1 b$ contains binding site for transcription factor SPI1 that regulates the expression of this gene, as well as $\mathrm{CpG}$, whose methylation level differs in the tissues studied. This CpG overlaps with the SPI1 binding site, which determines the differential expression of IL1B in humans. Altogether, our data confirm that the region between -2543 and $-2206 \mathrm{bp}$ is a part of the mouse $I l 1 \mathrm{~b}$ promoter/enhancer and determines its baseline expression level in various tissues.

\section{Disclosure Statement}

No competing financial interests exist.

\section{Funding Information}

The study was supported by a Presidential Grant for Young Scientists (project number MK-6016.2018.4) by Ministry of Science and Higher Education of the Russian Federation.

\section{Supplementary Material}

Supplementary Table S1

Supplementary Figure S1

Supplementary Figure S2

Supplementary Data S1

Supplementary Data S2

Supplementary Data S3

Supplementary Data S4

Supplementary Data S5

\section{References}

de Araújo-Souza, P.S., Hanschke, S.C.H., and Viola, J.P.B. (2015). Epigenetic control of interferon-gamma expression in CD8 T cells. J Immunol Res 2015, 849573.

Auron, P.E., Webb, A.C., Rosenwasser, L.J., Mucci, S.F., Rich, A., Wolff, S.M., et al. (1984). Nucleotide sequence of human monocyte interleukin 1 precursor cDNA. Proc Natl Acad Sci USA 81, 7907-7911.

Bigildeev, A., Shipounova, I., Lubkova, O., and Drize, N. (2012). IL-1beta as growth factor for hematopoietic microenvironment. Exp Hematol 40, S87 (abstract).

Bigildeev, A.E., Zezina, E.A., Shipounova, I.N., and Drize, N.J. (2015). Interleukin-1 beta enhances human multipotent mes- enchymal stromal cell proliferative potential and their ability to maintain hematopoietic precursor cells. Cytokine 71, 246-254. Bigildeev, A.E., Zezina, E.A., and Drize, N.J. (2017). The effects of interleukin-1 beta and gamma-quantum braking radiation on mesenchymal progenitor cells. Mol Biol 51, 393-403.

Bigildeev, A.E., Zhironkina, O.A., Lubkova, O.N., and Drize, N.J. (2013). Interleukin-1 beta is an irradiation-induced stromal growth factor. Cytokine 64, 131-137.

Chomczynski, P., and Sacchi, N. (2006). The single-step method of RNA isolation by acid guanidinium thiocyanatephenol-chloroform extraction: twenty-something years on. Nat Protoc 1, 581-585.

Clark, B.D., Collins, K.L., Gandy, M.S., Webb, A.C., and Auron, P.E. (1986). Genomic sequence for human prointerleukin 1 beta: possible evolution from a reverse transcribed prointerleukin 1 alpha gene. Nucleic Acids Res 14, 7897-7914.

Dinarello, C.A. (1996). Biologic basis for interleukin-1 in disease. Blood 87, 2095-2147.

Dinarello, C.A. (2013). Overview of the interleukin-1 family of ligands and receptors. Semin Immunol 25, 389-393.

Fitzpatrick, D.R., Shirley, K.M., McDonald, L.E., BielefeldtOhmann, H., Kay, G.F., and Kelso, A. (1998). Distinct methylation of the interferon gamma (IFN-gamma) and interleukin 3 (IL-3) genes in newly activated primary CD8+ T lymphocytes: regional IFN-gamma promoter demethylation and mRNA expression are heritable in CD44(high)CD8+ T cells. J Exp Med 188, 103-117.

Godambe, S.A., Chaplin, D.D., Takova, T., and Bellone, C.J. (1994a). Upstream NFIL-6-like site located within a DNase I hypersensitivity region mediates LPS-induced transcription of the murine interleukin-1 beta gene. J Immunol 153, 143-152.

Godambe, S.A., Chaplin, D.D., Takova, T., and Bellone, C.J. (1994b). An NFIL-6 sequence near the transcriptional initiation site is necessary for the lipopolysaccharide induction of murine interleukin-1 $\beta$. DNA Cell Biol 13, 561-569.

Godambe, S.A., Chaplin, D.D., Takova, T., and Bellone, C.J. (1995b). Molecular dissection of the murine IL-1 $\beta$ promoter. Am J Ther 2, 677-686.

Godambe, S.A., Chaplin, D.D., Takova, T., Read, L.M., and Bellone, C.J. (1995a). A novel cis-acting element required for lipopolysaccharide-induced transcription of the murine interleukin-1 beta gene. Mol Cell Biol 15, 112-119.

Gray, P.W., Glaister, D., Chen, E., Goeddel, D.V., and Pennica, D. (1986). Two interleukin 1 genes in the mouse: cloning and expression of the cDNA for murine interleukin 1 beta. J Immunol 137, 3644-3648.

Hashimoto, K., Oreffo, R.O.C., Gibson, M.B., Goldring, M.B., and Roach, H.I. (2009). DNA demethylation at specific $\mathrm{CpG}$ sites in the IL1B promoter in response to inflammatory cytokines in human articular chondrocytes. Arthritis Rheum 60, 3303-3313.

Hashimoto, K., Otero, M., Imagawa, K., de Andrés, M.C., Coico, J.M., Roach, H.I., et al. (2013). Regulated transcription of human matrix metalloproteinase 13 (MMP13) and interleukin-1 $\beta$ (IL1B) genes in chondrocytes depends on methylation of specific proximal promoter $\mathrm{CpG}$ sites. J Biol Chem 288, 10061-10072.

Ilnytskyy, Y., and Kovalchuk, O. (2011). Non-targeted radiation effects-an epigenetic connection. Mutat Res 714, 113125.

Johnston, C.J., Piedboeuf, B., Rubin, P., Williams, J.P., Baggs, R., and Finkelstein, J.N. (1996). Early and persistent alterations in the expression of interleukin-1 alpha, interleukin-1 
beta and tumor necrosis factor alpha mRNA levels in fibrosisresistant and sensitive mice after thoracic irradiation. Radiat Res 145, 762-767.

Jones, P.A. (2012). Functions of DNA methylation: islands, start sites, gene bodies and beyond. Nat Rev Genet 13, 484-492.

Kominato, Y., Galson, D., Waterman, W.R., Webb, A.C., and Auron, P.E. (1995). Monocyte expression of the human prointerleukin 1 beta gene (IL1B) is dependent on promoter sequences which bind the hematopoietic transcription factor Spi-1/PU.1. Mol Cell Biol 15, 58-68.

Kulakovskiy, I.V., Vorontsov, I.E., Yevshin, I.S., Soboleva, A.V., Kasianov, A.S., Ashoor, H., et al. (2016). HOCOMOCO: expansion and enhancement of the collection of transcription factor binding sites models. Nucleic Acids Res 44, D116-D125.

Linard, C., Ropenga, A., Vozenin-Brotons, M.C., Chapel, A., and Mathe, D. (2003). Abdominal irradiation increases inflammatory cytokine expression and activates NF-kappaB in rat ileal muscularis layer. Am J Physiol Gastrointest Liver Physiol 285, G556-G565.

Linard, C., Marquette, C., Mathieu, J., Pennequin, A., Clarençon, D., and Mathé, D. (2004). Acute induction of inflammatory cytokine expression after gamma-irradiation in the rat: effect of an NF-kappaB inhibitor. Int J Radiat Oncol Biol Phys 58, 427-434.

Pietras, E.M., Mirantes-Barbeito, C., Fong, S., Loeffler, D., Kovtonyuk, L.V., Zhang, S., et al. (2016). Chronic interleukin-1 exposure drives haematopoietic stem cells towards precocious myeloid differentiation at the expense of self-renewal. Nat Cell Biol 18, 607-618.

Sambrook, J., Fritsch, E.F., and Maniatis, T. (1989). Molecular Cloning: A Laboratory Manual (Cold Spring Harbor Laboratory Press, Cold Spring Harbor, NY).

Shirakawa, F., Saito, K., Bonagura, C.A., Galson, D.L., Fenton, M.J., Webb, A.C., et al. (1993a). The human prointerleukin 1 beta gene requires DNA sequences both proximal and distal to the transcription start site for tissue-specific induction. Mol Cell Biol 13, 1332-1344.

Telford, J.L., Macchia, G., Massone, A., Carinci, V., Palla, E., and Melli, M. (1986). The murine interleukin $1 \beta$ gene: structure and evolution. Nucleic Acids Res 14, 9955-9963.

Tsukada, J., Saito, K., Waterman, W.R., Webb, A.C., and Auron, P.E. (1994). Transcription factors NF-IL6 and CREB recognize a common essential site in the human prointerleukin 1 beta gene. Mol Cell Biol 14, 7285-7297.

Vandesompele, J., De Preter, K., Pattyn, F., Poppe, B., Van Roy, N., De Paepe, A., et al. (2002). Accurate normalization of real-time quantitative RT-PCR data by geometric averaging of multiple internal control genes. Genome Biol 3, RESEARCH0034.

Zhou, D., Yu, T., Chen, G., Brown, S.A., Yu, Z., Mattson, M.P., et al. (2001). Effects of NF-kappaB1 (p50) targeted gene disruption on ionizing radiation-induced NF-kappaB activation and TNFalpha, IL-1alpha, IL-1beta and IL-6 mRNA expression in vivo. Int J Radiat Biol 77, 763-772.

Address correspondence to: Alexey Bigildeev, PhD

Laboratory for Physiology of Hematopoiesis National Research Center for Hematology of the Ministry of Health of the Russian Federation Novii Zikovskii proezd, 4 Moscow 125167

Russia

E-mail: bigildeev.ae@gmail.com

Received for publication December 18, 2019; received in revised form January 23, 2020; accepted January 27, 2020. 